\title{
Lugano Classification Limited Stage Hodgkin Lymphoma AJCC v8
}

National Cancer Institute

\section{Source}

National Cancer Institute. Lugano Classification Limited Stage Hodgkin Lymphoma AJCC v8. NCI Thesaurus. Code C141169.

Limited stage Hodgkin lymphoma based on the Lugano classification criteria. It includes stages I, IE, II, and IIE. (from AJCC 8th Ed.) 